\title{
Microarray as high throughput tool for tyrosinase gene expression analysis
}

\begin{abstract}
Hyper pigmentary problems, including post inflammatory hyper pigmentation, solar lentigos, and melasma, occur widely in the human population and are thus of broad interest for control. Tyrosinase is the key enzyme of melanogenesis and it's over expression results in hyper pigmentation. Tyrosinase is not only liable to human pigmentation, but also responsible for the undesirable enzymatic browning of fruits and vegetables, which is of economic importance. So in order to get rid of the problem, inhibitors of the enzyme tyrosinase have been concerned. Advances in genomics and proteomics have opened up new possibilities for the rapid functional assignment and global characterization of proteins and DNA. Organized collection of molecules on arrays have provided a vigorous platform for rapid screening, lead discovery and eradication of molecular characterization. Nowadays, it is used for the discovery of tyrosinase inhibitor. It involves microarrays of immobilized distinct small molecules or DNA or RNA of cells treated with molecules to be tested and binding of tyrosinase proteins or TYR gene respectively. Compound is then screened and the respective compound is identified as a new small-molecule binder to tyrosinase and used it as potential tyrosinase inhibitor. Its actual binding and inhibitory effects on tyrosinase is also validated using an enzyme-based inhibition assay or other binding assays. As a rapidly maturing technology, high-throughput proteomic and genomic pave the way forward in the treatment of skin hyper pigmentation. Hence in the present review we have focused on the use of microarray technique over the conventional strategies for the elucidation of tyrosinase inhibitors.
\end{abstract}

Keywords: hyper pigmentation, tyrosinase, proteomics, microarray, immobilized
Volume 6 Issue 2 - 2017

\author{
Naima Parveen, Kamal Uddin Zaidi, Sharique \\ A Ali, Ayesha S Ali \\ Department of Biotechnology Pharmacology, People's \\ University, Bhanpur, India
}

\section{Correspondence: Kamal Uddin Zaidi, Biotechnology}

Pharmacology Laboratory and Immunology Laboratory People's University, Bhanpur, Bhopal, 462037 India,

Email zaidi.kama192@gmail.com

Received: August 10,2017 | Published: October 06, 2017

\begin{abstract}
Abbreviations: DHI, dihydroxyindole; DHICA, dihydroxyindole-2-carboxylic acid; ER, endoplasmic reticulum

\section{Introduction}

Since ancient times, a face with fair skin is considered as a sign of beauty and the demand for young, healthy, bright and fair skin has created a whitening cosmetics market. The color of human skin and hair established through a number of factors. Melanogenesis i.e. the biosynthesis of melanin is the major factor. It is a multistage process involving melanin synthesis in special organelles called melanosomes of melanocytes and melanin transport to keratinocytes. The actual color of the skin is determined by the distribution pattern of the melanin by the melanocytes. Tyrosinase is considered as key enzyme of melanogenesis as it plays a central role in biosynthetic pathway of melanin and catalyzes the first two steps: the hydroxylation of

effects Hence research is going on for the elucidation of tyrosinase inhibitors with less or no side effects. ${ }^{6,7}$ Traditionally, the molecules having antityrosinase activity have been elucidated by using different time consuming and labour intensive experiments. But, with the advancement in technology and the introduction of high throughput techniques make it easier for the biotechnologists to screen many compounds at a time for its inhibitory activity against tyrosinase. One of the techniques of high throughput screening of a molecule is microarray It is a powerful tool used for profiling gene expression, protein-protein and protein-ligand interaction in high throughput. ${ }^{8}$ So it is possible with microarray to screen various compounds as tyrosinase inhibitors. Besides traditional and manual approaches of elucidation and development of tyrosinase inhibitors, here in this review we will focus the importance of the use of high throughput techniques with special emphasis on microarray.
\end{abstract} monophenol to diphenol and the oxidation of diphenol to quinine. ${ }^{1-4}$ Abnormal deposition or irregular distribution of melanin causes hyper pigmentary disorders such as melasma, freckles and age spots. One of the major causes of hyper pigmentation is probably UV light. However, the skin darkening can be restrained, at least to some extent, by deactivating the enzyme tyrosinase. ${ }^{5}$ So, in order to treat hyper pigmentation and the quest for skin whitening move forward the scientists of all over the world to search inhibitors of enzyme tyrosinase.

Although a number of ingredients from natural sources with an inhibitory effect on melanogenesis have been studied in order to treat hyper pigmentation as well as developed as cosmetic agents to make skin white. But many of them find associated with several side

\section{Melanogenic enzyme tyrosinase and its mechanism}

Tyrosinase, a copper containing enzyme is the key enzyme in melanogenesis. It catalyzes the first step of melanin synthesis. Tyrosinase is responsible for the conversion of tyrosine to L-DOPA and DOPA quinone. After DOPA quinone formation by tyrosinase, the compound is converted into cysteinyl DOPA (in the presence of cystein) and DOPA chrome (in the absence of cystein) through autooxidation. These compounds are further converted into different melanins through a series of enzymatic and spontaneous reactions. Eumelanin is formed through reactions from dihydroxyindole (DHI) and dihydroxyindole-2-carboxylic acid (DHICA) which are the reaction products of DOPA chrome (Figure 1). Subsequently pheomelanin is formed by the oxidative polymerization of cysteinyl 
DOPA. ${ }^{9-11}$ Though there are three enzymes involved in melanogenesis pathway, only tyrosinase is extremely important for melanogenesis, as it plays a key role in the process. Tyrosinase is a glycoprotein positioned in melanosome membrane with an inner melanosomal domain that holds the catalytic region, followed by a short transmembrane domain and a cytoplasmic domain. The remarkable characteristic observed in tyrosinase is that a central copper-binding domain is conserved, which contains strictly conserved amino acid residues, including three histidines. Tyrosinase is produced only by melanocytic cells. It is synthesized and subsequently processed in endoplasmic reticulum (ER) and Golgi bodies, and then it gets trafficked to melanosomes, in which the pigment melanin is formed. ${ }^{12-16}$

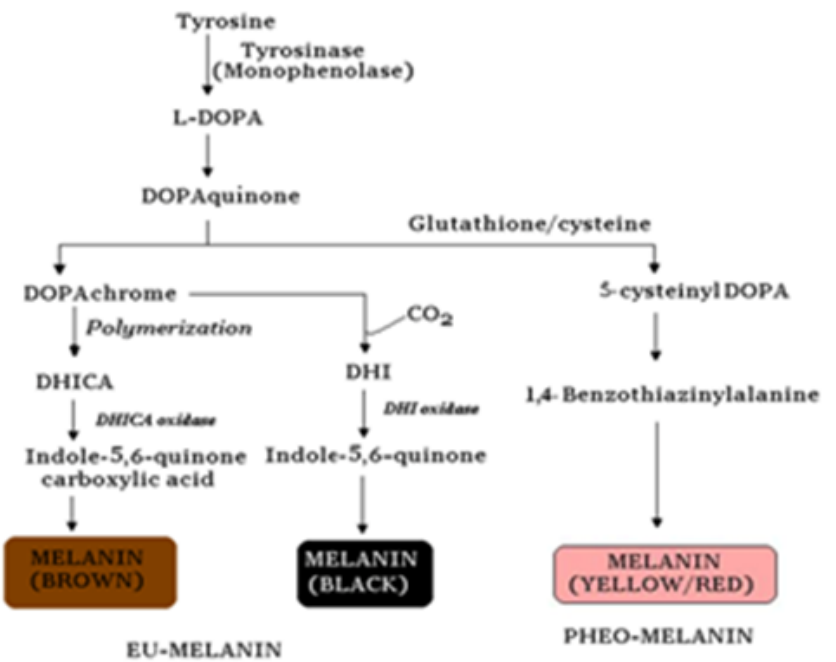

Figure I Production of different pigments by the action of tyrosinase.

Different assays implemented for the analysis of change in tyrosinase gene expression

Western blot: In this method mixture of protein is first separated on the basis of molecular mass and charge by SDS Polyacrylamide gel electrophoresis, then transferred on nitrocellulose membrane and probed with antibody specific to protein of interest. Western blot is used in various studies which utilize expression profile of tyrosinase and other melanogenic enzymes to find out potent antimelanogenic agent. Oh et al., ${ }^{17}$ demonstrated reduced expression of tyrosinase and MITF gene by Ficus deltoidea extract through western blotting. Extracts of citrus press cakes, Oreocnide fruticosa and Agaricus bisporus were examined for their mealanogenic and antimelanogenic activity in terms of expression of melanogenic enzymes including tyrosinase by using western blot analysis. ${ }^{18-20}$ Similar work was done by Lee et al., ${ }^{21}$ using Flavonoid Glycosides from Limonium tetragonum as antimelanogenic agent. Despite of its application it has certain limitations such as it can detect only denatured protein, relatively technically complex, requires many steps and large quantity of sample, time consuming and expensive.

ELISA: ELISA is a quantitative assay used to detect native protein and protein interactions. Antimelanogenic activity of chrysin was tested by assessment of tyrosinase inhibition activity in B16 cells using ELISA method. ${ }^{22}$ Although ELISA assays are technically less difficult than western blot and can be adapted to high throughput with automated detection system. However these are not specific and can give false positive results due to cross reactivity of the detective antibody with other protein in the sample.
PCR: Quantitative PCR or Real time PCR has been widely used for the quantitative measurement or detection of alteration in gene expression levels in response to specific pharmacological agent. Inhibitory effect of arctigenin from Fructus arctii extract on tyrosinase gene expression was analysed by Park et al. ${ }^{23}$ using real time qPCR. Similarly Lee et al., ${ }^{24}$ elucidate the inhibitory effect of 4-n-butylresorcinol on tyrosinase mRNA expression in $\mathrm{B} 16 \mathrm{~F} 10$ melanoma cells through real time PCR. Reverse transcription PCR have also been extensively used for estimation of gene expression. It has been found that aglycone of Rh4 inhibits melanin synthesis via decrease in tyrosinase and MITF gene expression which was analysed using reverses transcription PCR. ${ }^{25}$ Though it has been widely used for gene expression analysis, there are certain troubles found associated while accessing with PCR like difficulty in designing correct probes and primers, testing of their efficacy require more time and efforts.

\section{Microarrays}

More recently high throughput techniques have been developed for the study of biomolecules including protein microarray and DNA microarray. Protein microarrays are basically of three types which are currently been used to study biochemical activities of protein: Analytical microarray, Functional microarray, and Reverse phase microarray. Analytical microarrays are normally used to measure binding affinities, specificities, and protein expression levels in the mixture of protein. Functional protein microarrays are composed of full length proteins or domains. It is used to study protein interactions. Reverse phase microarrays are prepared by isolating cells from various tissues and get it lysed. These lysates are immobilized on to the slides. The slides are then probed with antibodies against protein of interest. ${ }^{26,27}$ Small molecule microarrays are another powerful high throughput tool for gathering information about direct interactions between protein of interest and small molecule. It is being used to detect ligands for new drug discovery. Small molecules including organic compounds, natural products, peptides have been immobilized on microarray slides and a variety of biomolecules can be incubated with these slides, ${ }^{28,29}$ (Figure 2).

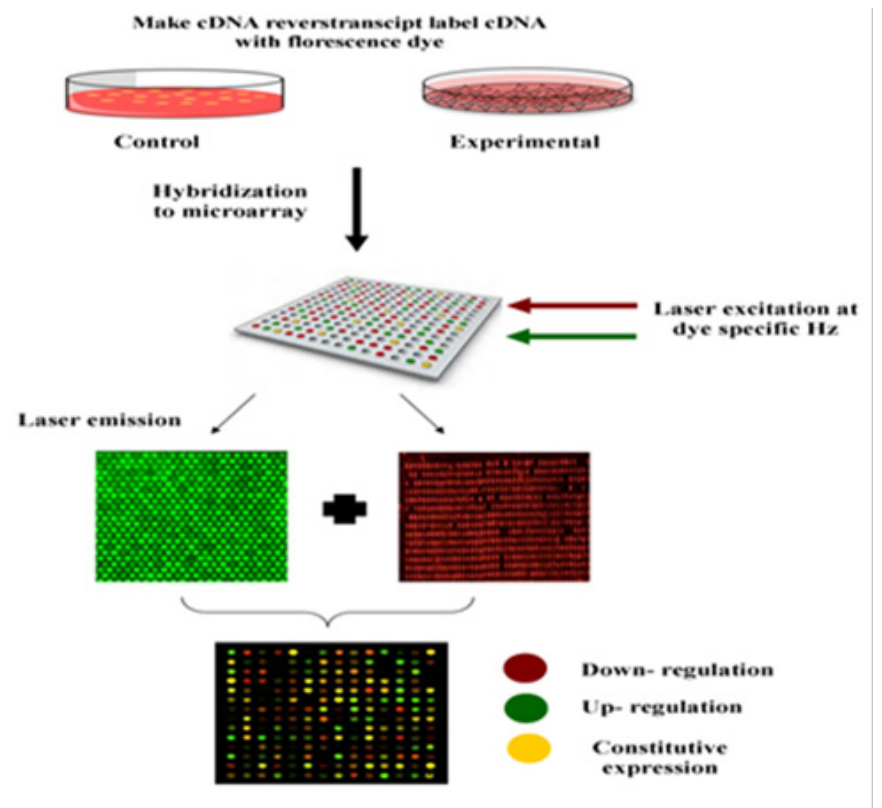

Figure 2 Schematic representation of cDNA microarray assays for gene expression. 


\section{Microarrays for the elucidation of decreased expression of melanin synthesis gene including TYR gene by the action of certain compounds}

In 2010 Choi et al., ${ }^{30}$ established melanoma cell line named primary culture of melanoma cell derived from lymph node (PML-1). Through DNA microarray and two dimensional matrix assisted laser desorption ionization time of flight mass spectrometry analysis they have found that PML-1 decreased the expression of melanogenesis markers including TYR, MITF, and TRP-1 genes. In another study, gene expression profiling of three well known skin lightener was illustrated by Gruber $^{31}$ using DNA microarray. Human epidermal keratinocyte were treated with variable ratios of hydroquinone, kojic acid, and niacinamide. After 24hours of treatment, total RNA was extracted out from the culture, amplified and labeled them with fluorescent dyes. The fluorescently labeled RNA was applied to the DNA microarray chips having TYR, COX1, and FTH1, MC1R gene immobilized on it and the chips were hybridized overnight. Results of their experiment confirmed that all three compounds show decreased expression of three genes i.e. TYR, COX1, and FTH1 but have little effect on MC1R gene.

Small molecule microarray system is utilized for examining the binding efficiencies between tyrosinase and small molecules in order to discover novel tyrosinase inhibitors. In this area, very interesting work was done by Lee $\mathrm{HY}^{32}$ as most of the known tyrosinase inhibitors are natural polyphenols, so they used natural products for screening approach with some other synthetically synthesized small molecules. They have tested several surface modification methods for glass slides and tested many compounds for their binding affinity with tyrosinase, and ultimately identified 2,4,4- trihydroxychalcone as a new small molecule binder to tyrosinase so it can be used as tyrosinase inhibitor.

\section{Conclusion}

Neither over production of melanin in human skin nor enzymatic browning of fruits and vegetables is required. Tyrosinase is the key enzyme catalyzes the rate limiting step of melanogenesis as well as browning of fruits and vegetables. So, quest for the inhibitors of tyrosinase have been of great concern. At present technology has driven to deliver several methods that allow monitoring of alteration in the expression of tyrosinase by the action of certain compounds which will be further used as tyrosinase inhibitors. Although there are several assays utilized for analysis of tyrosinase gene expression. Those are time consuming, technically complex and not highly specific. Protein, DNA and small molecule microarrays are thus potentially powerful tools to overcome this problem. They provide a platform to profile DNA-DNA, protein-protein, protein-ligand interactions and gene expression in high throughput. They have lead to the trafficking of large number of molecules at a time, for testing of tyrosinase inhibitors. These are rapid, automated, and highly sensitive and time saving methods. Future research with the aid of high throughput analysis in search of potential tyrosinase inhibitors makes easier for the pharmacist and cosmetician to treat skin hyper pigmentation.

\section{Acknowledgements}

The authors extend heartfelt appreciation to the Secretary and Principal of Saifia College of Science, Bhopal, India, for encouragement.

\section{Conflict of interest}

The authors declare that there is no conflict of interests regarding the publication of this paper.

\section{References}

1. Costin GE, Hearing VJ. Human skin pigmentation: melanocytes modulate skin color in response to stress. FASEB J. 2007;21(4):976-994.

2. Zaidi KU, Ali AS, Ali SA. Purification and Characterization of Melanogenic Enzyme Tyrosinase from Button Mushroom. Enzyme Research. 2014;(2014):1-6.

3. Ali S and Naaz I. Current challenges in understanding the story of skin pigmentation: Bridging the morphoanatomical and functional aspects of mammalian melanocytes. In: Muscle cells and Tissue. Europe: In tech publications; 2015.

4. Abbas Q, Raza H, Hassan M, et al. Acetazolamide inhibits the level of tyrosinase and melanin: An enzyme kinetic, in vitro, in vivo and in silico studies. Chem Biodivers. 2017:14(9).

5. Bell AA, Wheeler MH. Biosynthesis and functions of fungal melanins. Annu Rev Phytopathol. 1986;24:411-451.

6. Chang TS. An updated review on tyrosinase inhibitors. Int $J$ Mol Sci. 2009;10(6):2400-2475.

7. Gillbro JM, Olsson MJ. The melanogenesis and mechanisms of skinlightening agents-existing and new approaches. Int J Cosmet Sci. 2011;33(3):210-221.

8. Sun H, Chen GY, Yao SQ. Recent advances in microarray technologies for proteomics. Chem Biol. 2013;20(5):685-699.

9. Prota G. The chemistry of melanins and melanogenesis. Fortsch Chem Organ Nature. 1995;64:93-148.

10. Zaidi KU, Ali AS, Ali SA, et al. Microbial Tyrosinases: Promising Enzymes for Pharmaceutical, Food Bioprocessing, and Environmental Industry. Biochemistry Research International. 2014;2014:1-16.

11. Zaidi KU, Ali AS, Ali SA. Effect of Purified Mushroom Tyrosinase on Melanin Content and Melanogenic Protein Expression. Biotechnology Research International. 2016;2016:1-8.

12. Kwon BS, Haq AK, Pomerantz SH, et al. Isolation and sequence of a cDNA clone for human tyrosinase that maps at the mouse c-albino locus. Proc Natl Acad Sci USA. 1987;84(21):7473-7477.

13. Sánchez Ferrer A, Rodrígue López JN, García Cánovas F, et al. Tyrosinase: A comprehensive review of its mechanism. Biochim Biophys Acta. 1995;1247(1):1-11.

14. Kim YJ and Uyama H. Tyrosinase inhibitors from natural and synthetic sources: Structure, inhibition mechanism and perspective for the future. Cell Mol Life Sci. 2005;62(15):1707-1723.

15. Khan MT. Novel tyrosinase inhibitors from natural resources - their computational studies. Curr Med Chem. 2012;19(14):2262-2272.

16. Jia YL, Zheng J, Yu F, et al. Anti-tyrosinase kinetics and antibacterial process of caffeic acid N-nonyl ester in Chinese Olive (Canarium album) postharvest. Int J Biol Macromol. 2016;91:486-495.

17. Oh MJ, Hamid MA, Ngadiran S, et al. Ficus deltoidea (Mas cotek) extract exerted anti-melanogenic activity by preventing tyrosinase activity in vitro and by suppressing tyrosinase gene expression in B16F1 melanoma cells. Arch Dermatol Res. 2011;303(3):161-170.

18. Kim SS, Kim MJ, Choi YH, et al. Down-regulation of tyrosinase, TRP1, TRP-2 and MITF expressions by citrus press-cakes in murine B16 F10 melanoma. Asian Pac J Trop Biomed. 2013;3(8):617-622.

19. Han JH, Byeon SH, Hyun CG, et al. Melanogenesis Inhibitory Activity in the Extracts of Oreocnide fruticosa (Gaudich.) Hand.-Mazz. Branches. Journal of Applied Pharmaceutical Science. 2014;4(01):166-169.

20. Zaidi KU, Ali AS, Ali SA. Melanogenic effect of purified mushroom tyrosinase on B16F10 Melanocytes: A Phase Contrast and Immunofluorescence Microscopic Study. Journal of Microscopy and Ultra structure. 2016;5(2):82-89. 
21. Lee SG, Karadeniz F, Seo Y, et al. Anti-Melanogenic Effects of Flavonoid Glycosides from Limonium tetragonum (Thunb.) Bullock via Inhibition of Tyrosinase and Tyrosinase-Related Proteins. Molecules. 2017;22(9):E1480

22. Zhu L, Lu Y, Yu WG, et al. Anti-photoageing and anti-melanogenesis activities of chrysin. Pharm Biol. 2016;54(11):2692-2700.

23. Park H, Song KH, Jung PM, et al. Inhibitory Effect of Arctigenin from Fructus Arctii Extract on Melanin Synthesis via Repression of Tyrosinase Expression. Evid Based Complement Alternat Med. 2013;2013:1-10.

24. Lee SJ, Son YH, Lee KB, et al. 4-n-butylresorcinol enhances proteolytic degradation of tyrosinase in B16F10 melanoma cells. Int J Cosmet Sci. 2017;39(3):248-255.

25. Jeong YM, Oh WK, Tran TL, et al. Aglycone of Rh4 inhibits melanin synthesis in B16 melanoma cells: Possible involvement of the protein kinase A pathway. Biosci Biotechnol Biochem. 2013;77(1):119-125.

26. Zhu H, Bilgin M, Bangham R, et al. Global analysis of protein activities using proteome chips. Science. 2001;293(5537):2101-2105.
27. Hall DA, Zhu H, Zhu X, et al. Regulation of gene expression by a metabolic enzyme. Science. 2004;306(5695):482-484.

28. Duffner JL, Clemons PA, Koehler AN. A pipeline for ligand discovery using small-molecule microarrays. Curr Opin Chem Biol. 2007;11(1):74-82.

29. Kasalena DE, Wassaf D, Koehler AN. Ligand discovery using smallmolecule microarrays. Methods Mol Biol. 2012;803:249-263.

30. Choi TY, Sohn KC, Kim JH, et al. Impact of $\mathrm{NAD}(\mathrm{P}) \mathrm{H}$ : quinone oxidoreductase-1 on pigmentation. J Invest Dermatol. 2010;130(3):784 792.

31. Gruber JV, Holtz R. Examining the Impact of Skin Lighteners in vitro. Oxid Med Cell Longev. 2013;2013:1-7.

32. Lee HY, Park SB. Surface modification for small-molecule microarrays and its application to the discovery of a tyrosinase inhibitor. Mol Biosyst. 2011;7(2):304-310. 